\title{
ZEEHC: Zone-Based Energy Efficient Hierarchal Clustering Hierarchy for Wireless Sensor Networks
}

\author{
Nitin Mittal*1 \\ ${ }^{1}$ Department of Electronics and Communication, Chandigarh University, Gharuan-140413, Punjab, India
}

Article History: Received: 11 January 2021; Accepted: 27 February 2021; Published online: 5 April 2021

Abstract- Wireless Sensor Network (WSN) is an emerging technology with potential applications in the field of
habitat monitoring and industrial applications. Sensors monitor changes in an environment's physical attribute
such as temperature, and observe the data collected and forward it to the base station (BS). Mostly these sensors
are unattended, and their limited battery life makes energy a valuable resource that has to be used wisely. For the
collection of information, the sensor network must be maintained for a longer duration of time in an energy-
efficient manner.Therefore, designing protocols that prolong the life of the network and which are energy-
efficient is incessantly fascinating. This paper proposed a protocol referred to as the zone-based energy-efficient
hierarchical clustering (ZEEHC) protocol that divides the network into small zones and increases network
lifetime. In order to achieve minimum energy consumption, multi-hop contact is implemented between ZHs -
CHs -BS. The results further reveal that the proposed protocol significantly outperforms existing algorithms in
terms of energy optimization and system lifetime.
Keywords- Clustering, Residual energy,SEECH, ZEEHC, Network lifetime, WSN.

\section{Introduction}

WSN is basically a group of wireless nodes that have limited energy capabilities and are placed at random on an energetically varying environment. The selection of routing strategies is a vital problem for delivering the packets to the sink effectively. In addition, the routing strategy implemented in these networks must ensure the minimum energy consumption to optimize the lifetime of the network[1].

The problems of the network lifetime and availability of data are crucial in WSN because they are deployed in an unsympathetic environment[2]. The operation of the sensor network takes place in different stages such as planning, deployment and operation and at last action[3].The planning stage consists of conducting of survey of the site in order to examine the environment and the circumstances for the selection procedure in the implementation mechanisms. Deployment stage consisting of the randomly deployment of the sensors in the sensing area. Following the deployment stage require sensor network administrator for the evaluation purpose where sensors to approach more coverage. Operation stage includes proper functioning of maintaining projects, sensors inspect environment and provide information. Post operative stage includes powering off and safeguards sensors for upcoming functions and to destroy sensor network.

The military and defense industries implemented the first WSN in the mid-70s. WSN is used during the Vietnam War to bear enemy detection in remote areas.But their implementations had many drawbacks including the large sensor size and higher energy utilization and the limited network capability.

Routing protocols are one of the key technologies in the WSN. Routing is a complete challenge in WSN [4], due to its intrinsic characteristics. Clustering is a well-know and generally used tentative data transmission technique, and is mainly useful for such applications that require scalability [5]-[10]. In clustering protocols, the entire network is subdivided into clusters having cluster members (CMs) and one cluster head (CH). All CMs transmit their sensed information to $\mathrm{CH}$, which is responsible for long run data transmission to the sink. The energy consumption using clustering approach is much lower than the direct transmission. However, in clustering due to overburden at the $\mathrm{CH}$ due to long transmission of data, $\mathrm{CHs}$ die out earlier[11].

In this paper, we propose a Zone based Energy Efficient hierarchal Clustering (ZEEHC) protocol to maintain the energy utilization among all nodes. The WSN is divided into equal size of zone. ZEEHC helps to improve the network lifetime with low utilization of energy in the WSNs. The clustering protocols are actually contains cross layering techniques for scheming energy efficient hierarchal WSNs [12] where the nodes that belong to a $\mathrm{CH}$ gives their data to the $\mathrm{CH}$ and then $\mathrm{CH}$ gives aggregate data to the relay node in the zone. The aggregated data is then transmitted to BS by relay node using multi-hop communication. The clustering schemes can raise network lifetime and better energy efficiency by decreasing all of the energy consumption and maintaining utilization belonging the nodes during the network lifetime [13], [14]. The clustering protocols are explained according to the schemes they accept to select $\mathrm{CHs}$ as well as communicating the aggregate information to the sink[15], [16]. 


\section{RELATED WORK}

Energy efficient design and the latest wireless technology for wireless devices, described by Heinzelman et.al[17]. Various types of applications covering a large area, along with real-time cascade audio and video distribution, to transfer large amounts of data from one device to another. The above applications containgreat achievement in the ring, it often endure from ability restrain. Wireless devices often have limited intensity resources, because the nodes are battery operated. This makes the program with limited bandwidth by making a transmission error prone. WSN subsist of a set of sensor nodes empower the control of an assortment of settings for applications. In WSNs, it is very relevant for sensor network for a long time. In this case, integration of data useful to diminish the bulk of information transferred among nodes of sensors and BS[18], [19].

There are many different types of energy efficient clustering protocols designed among cluster based network frame. The LEACH[11] protocol defines energy utilization is same in all the nodes during selection of cluster heads. In this protocol,the non-cluster heads gives their data to nearest $\mathrm{CH}$ and $\mathrm{CH}$ forwards the aggregated packet to sink. In LEACH protocol, $\mathrm{CH}$ decision basically depend on the percentage of $\mathrm{CHs}$ for the network (resolute on priori) and the number of time that node has been $\mathrm{CH}$ so far. This evaluation is taken by the node $n$ by selecting a random number between 0 and 1 .If the selected number is less than a threshold $T(n)$, the node become $\mathrm{Ch}$ for the current round. The Threshold is as:

$$
T(n)=\left\{\begin{array}{l}
\frac{p}{(1-p *(\operatorname{rmod} 1 / p))} \text { if } n \in G \\
0 \quad \text { otherwise }
\end{array}\right.
$$

The $\mathrm{CH}$ forwards the accumulated data to the sink or destination. LEACH incorporate random shot orbit of high-intensity $\mathrm{CH}$ location with the sensor to escape exhausting the lifetime any sensors in the grid[20], [21]. The problem is that when the data collection process does not give the size reduction outgoing data, clustering can propose additional power utilization and a lag in the information assemble process[22]. LEACH works well with fully fusible and partly fusible data. But, more emphasis is made on energy consumption and collection efficiency is neglected.

The HEED protocol using a multi-hop communication scheme in which $\mathrm{CH}$ selects according to the residual energy as well as lower power stage required by a node to transfer the information with its $\mathrm{CH}$ and $\mathrm{CH}$ gives their data to base station [23].

In EECS, aggressive allotment of clusters takes place which is established on cluster distance from the main station. The conclusion is an innovation that dwelling the obstacle that clusters at a highest distance from the sink lack more power for communication than those that are nearest. Basically it contribute same dissemination of power in the networks, appear in network lifetime. SEECH is a scalable energy efficient clustering hierarchical protocol which employs a hierarchal clustering. In SEECH, divide area into three regions as well as all the nodes are randomly deployed in a given regions. After that we calculate degree of nodes(degree means distance between each node according to region).If the probability of tentative cluster head $\left(P_{c i}\right)$ and relay head $\left(P_{r i}\right)$ is less than random value on the basis of degree of node can be selected as tentative $\mathrm{CH}$ and relay head. If the probability of tentative $\mathrm{CH}$ and relay head is greater than random value than calculate the score of all nodes (score is defined as product of distance of all the nodes from their respective cluster head and relay head) and nodes having minimum score select as actual cluster head and relay head. SEECH is a scalable energy efficient clustering hierarchical protocol which employs a hierarchal clustering. In SEECH, divide area into three regions as well as all the nodes are randomly deployed in a given regions. After that we calculate degree of nodes(degree means distance between each node according to region).If the probability of tentative cluster head $\left(P_{c i}\right)$ and relay head $\left(P_{r i}\right)$ is less than random value on the basis of degree of node, thenthese are selected as tentative $\mathrm{CH}$ and relay head. If the probability of tentative $\mathrm{CH}$ and relay head is greater than random value than calculate the score of all nodes (score is defined as product of distance of all the nodes from their respective cluster head and relay head) and nodes having minimum score select as actual cluster head and relay head.

$$
\begin{aligned}
& P_{c i}=\left\{\begin{array}{cc}
\left(\left(E_{\text {resi }} \times \operatorname{deg} i\right) / p c-\text { tot }\right), & \text { Eresi } \geq \operatorname{Eav}(1-\lambda) \\
0, & \text { otherwise }
\end{array}\right. \\
& p c-\text { tot }=\left(\left(\text { Eav } \times \sum N \operatorname{deg} i\right) / 2 K_{C H}\right) \\
& \begin{array}{c}
P_{r i}=\left\{\begin{array}{c}
\left(\left(E_{\text {resi }} \times \operatorname{deg} i\right) / p r-t o t\right), \quad \text { Eresi } \geq \operatorname{Eav}(1-\lambda) \\
0, \quad \text { otherwise } \\
p r-t o t=\left(\left(\text { Eav } \times \sum N(1-\operatorname{deg} i)\right) / 2 K R\right)
\end{array}\right.
\end{array}
\end{aligned}
$$




\section{Energy Dissipation Radio Model}

The first form of radio model for WSNs is used which is presentedin Fig.1.Only the free space canal model is used for the experiments described here. Thus the radio expends energy to transmit $l$-bit message a distance $d$ :

$$
\begin{array}{rl}
E_{T X}(k, d)=k & * E_{\text {elec }}+k * E_{r s} * d^{2} d<d_{o} \\
k & * E_{\text {elec }}+k * E_{\text {amp }} * d^{4} d \geq d_{o}(7)
\end{array}
$$

The first term represents the power consumption radio distribution, while the other represents the energy consumption for extreme Radio model. Appropriate space $E_{f s}$ and multi-path fading $E_{a m p}$ channel model depends on transmission distance. When receiving this data, radio spend

$$
E_{R x}(k)=\mathrm{k}^{*} \text { Eelec }
$$

In addition, the operation aggregation of data will absorbenergy $E_{D A}$.

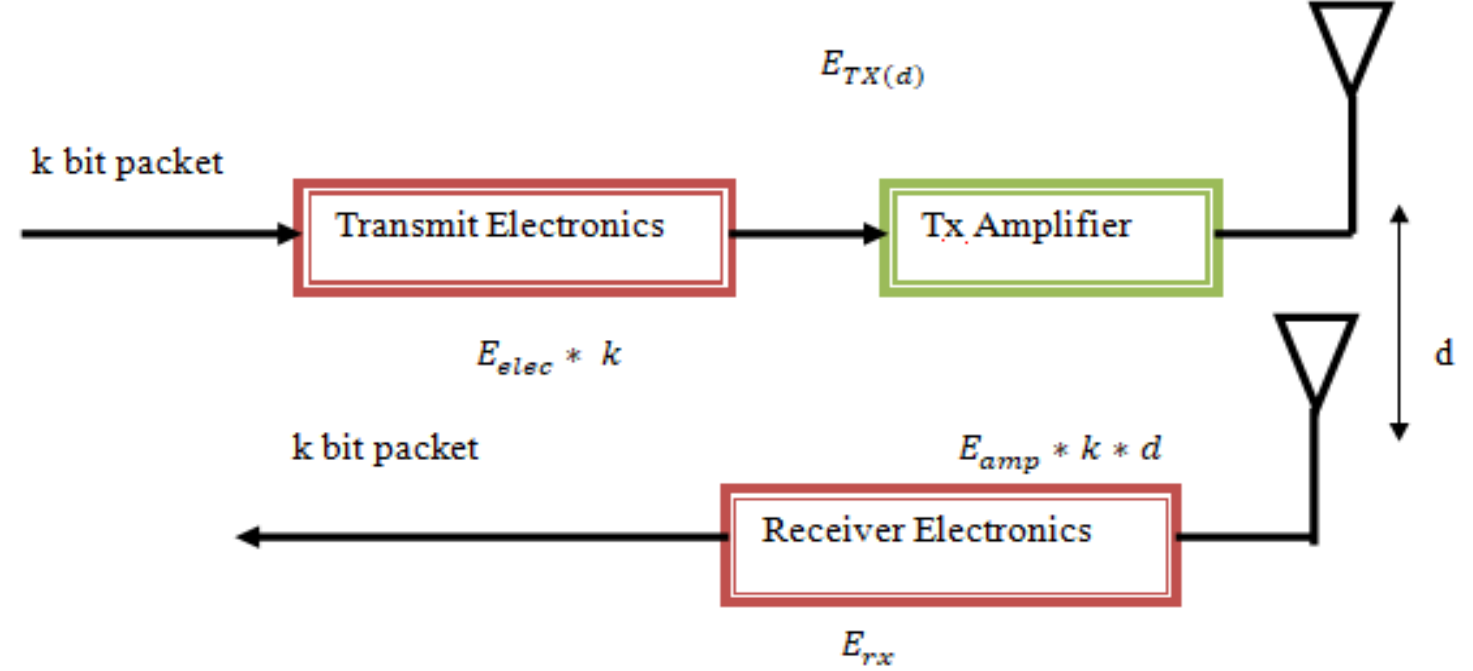

Fig1 First order radio energy model [16]

\section{ZEEHC PROTOCOL}

This work is motivated by EEHC protocol which is extended to an enhanced version of EECH. Basically, in this work a new zone based scheme introduced based EEHC protocol is introduced for WSNs to increase network lifetime and stability period. In LEACH protocol, $\mathrm{CHs}$ get changed in every round and after cluster formation, those $\mathrm{CHs}$ don't get chance to become $\mathrm{CHs}$ for next $1 / \mathrm{p}$ rounds. In our proposed work, we introducing modified EEHC as $\mathrm{CH}$ selection technique in the presence of Zone. It introduces combined threshold parameter in cluster formation for $\mathrm{CH}$ election in every upcoming round. This is how we can store more energy consumption on $\mathrm{CH}$ election and cluster formation processes.

In ZEEHC, area divided into zone and distributing the nodes in network area. Basically, we present a technique to reduce the energy dissipation and prolong life of the network. Therefore, the subsequent changes have made in the SEECH protocol to improve the adaptability and better the network lifetime.

Model Architecture and Basic Assumptions

- $\quad$ Number of nodes is $100,400,1000$ in the network for three scenarios.

- Homogenous network consist by WSN.

- The BS is situated outside WSN.

- Sensor nodes have same initial energy.

- All nodes can forward data to ZH.

- Data compression is done by ZH.

- In first node, exclusive node has probability $p$ of becoming the ZH.

- Nodes with higher energy in previous round become tentative relay.

- Nodes which have minimum distance in previous round become RH.

\section{ZEEHC Process}

First of all network parameters are initialized. As the network consists of homogeneous sensors, all have the same initial energy before that sensor field is decided. After the deployment of sensors, rounds are started. In a given region $\mathrm{ZH}$ and $\mathrm{RH}$ selection occur on the basis of remaining energy of nodes in previous round and 
distance from the BS. Then cluster member sends their data to the nearest $\mathrm{ZH}$, data aggregated by $\mathrm{ZH}$ and forwarded to RH by using multi-hopping communication and then send to BS.

\section{Zone Head Selection}

In ZEEHC protocol, area is divided into number of zones and sensors are deployed randomly in a given network. Generally nodes having high energy are more appropriated selected for $\mathrm{CH}$. The advantages of this technique is that high amount of nodes might be covered by less number of $\mathrm{CH}$ utilize low power data transmission. The method of selection of $\mathrm{CH}$ as follows. Firstly, normal node checks the maximum energy of previous node in previous round for new node and also identifies the minimum distance from BS is selected as a $\mathrm{CH}$.

\section{Relay Head Selection}

The nodes nearer to the BS can helpful to reduce data communication cost. The protocol selects these nodes as $\mathrm{CHs}$ so that energy is stored for information transfer to the BS. The nodes having larger intra-cluster energy utilization those nodes cannot be a $\mathrm{CH}$. For maintaining energy utilization, large amount of energy utilization tasks should be given to exclusive node during network lifetime. Since, in this protocol relay nodes are selected on the basis of energy and distance from BS. Firstly, in previous round energy of new node is high than the energy of previous node and distance of new node is less than the previous node from BS mainly selected as relay node.

In ZEEHC nodes are randomly deployed and in exclusive zone one $\mathrm{ZH}$ and one relay node is selected based on remaining energy and the separation from BS. In ZEEHC multi-hopping scheme also used for the data transmission to sink. Let it to be taking a particular threshold value if the energy of $\mathrm{CH}$ is higher than the threshold value then $\mathrm{CH}$ node directly transmit the data to the $\mathrm{BS}$. If the energy of $\mathrm{CH}$ is less than the threshold value then it firstly transmits the data to the nearest relay to next relay, so multi-hopping is done during this proposed scheme.

Algorithm 1: Zone Head Selection Algorithm

1. No. of zones $=$ n

2. Divide into for equal zone

3. for (zone $=$;zone $>=n$; zone ++ )

4. Deploy sensor node each Zone

5. end for

6. While (normal node $=$ dead node)

7. // Relay selection

8. For $($ zone $=$;zone $>=4$;zone ++$)$

9. If (check node for Maxi. Energy in Previous node)

10. Tentative relay node New Node

11. If (New node Distance from B.S is mini. Previous round)

12. Relay Node New Node

13. end if

14. end if

15. // Selection of Zone head

16. If (Energy of New node $=$ max. energy of Previous round $)$

17. Normal Node $\neq$ relay Node ;

18. If (New node Distance from B.S < Previous $\mathrm{ZH}$ node Distance from BS )

19. Normal Node Hew Node ;

20. end if

21. end if

22. end while

23. // ZH aggregate data sends direct from Node to the data and send to zone relay node

24. Relay node tx. data to BS using multi-hopping to other relays.

25. // check for alive and dead node

26. If(Energy of node $<0$ )

27. Dead node $=$ dead node +1 ;

28. end if

29. Total Alive node $=$ Alive node - dead; 


\section{Proposed Algorithm}

The flowchart for the operation of ZEEHC is shown in Fig. 2. There are five phases which describes the process of our proposed scheme ZEEHC. In this, each round repeats periodically having differentphases, as follows:

1. Initialization phase: In the first phase, there are two steps involved. First step is to initialize network parameters. Network parameters are area, rounds, nodes etc are initialized. Second step is to deploy the sensors randomly in the given area.

2. Statistics phase: This phase gives information about the dead nodes, alive nodes, first node dead, average node dead node dead, last node dead etc.

3. Selection phase:In this phase,ZH and RH is selected on the basis of some sensor node concepts like residual energy, distance from $\mathrm{BS}$, distance between node to $\mathrm{ZH}$, connection time and the number of rounds that the node $\mathrm{n}$ was selected $\mathrm{ZH}$ so far.

4. Association phase: After selection of the ZHs in the current round, once each non $\mathrm{ZH}$ receives the information from the $\mathrm{ZHs}$, it determine its cluster by choosing one of the ZHs with minimum distance. In this way, nodes get associated with their corresponding CHs.

5. Energy consumption phase: In order to calculate energy dissipation, the first order radio model is used and its parameters are used. The energy consumed during node to $\mathrm{ZH}$ communication, $\mathrm{ZH}$ transmission and reception are being calculated.

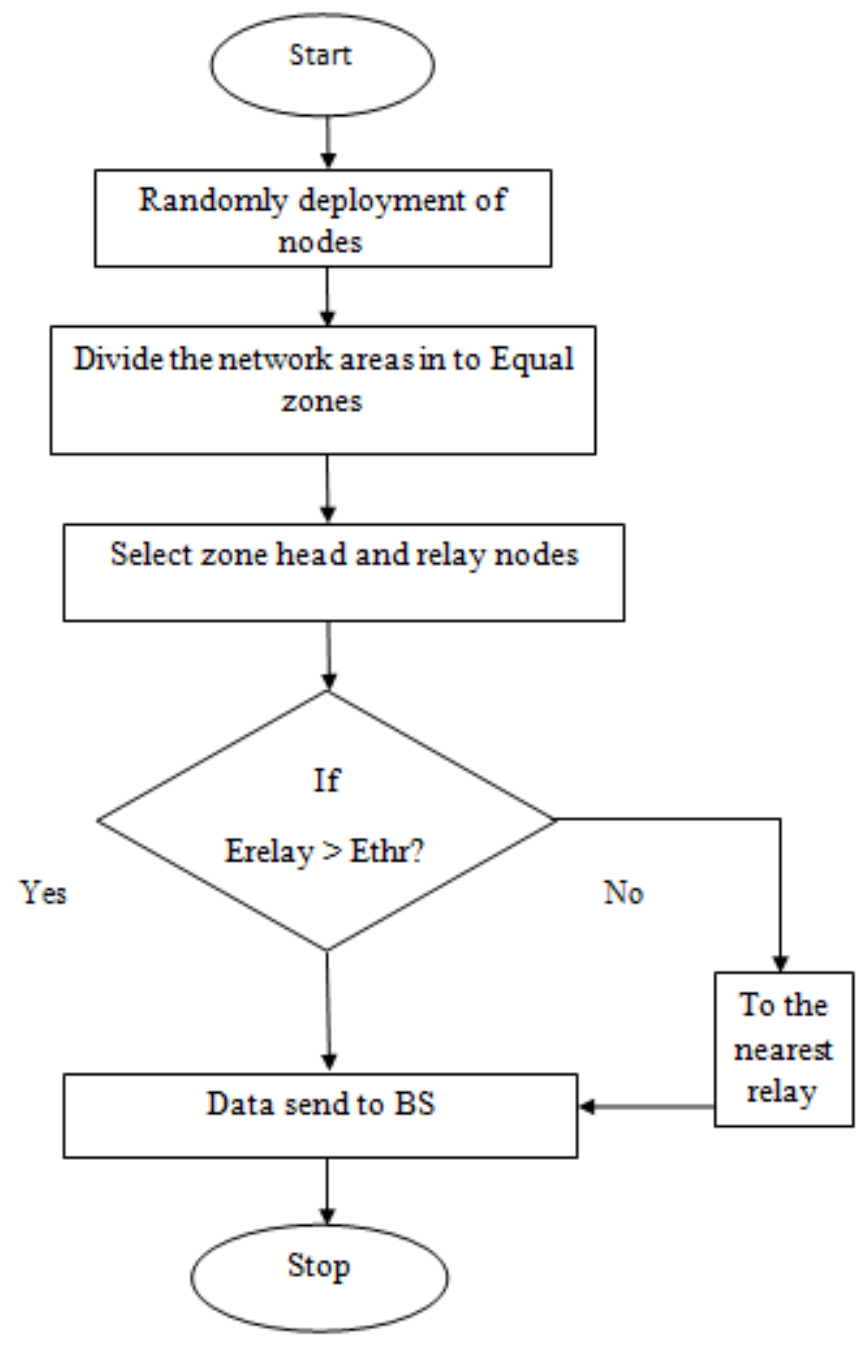

Fig. 2Flowchart for the operation of ZEEHC

\section{V. Results and Discussions}

Energy efficient deployment is not an easy task due to the large number of parametersi.e.energy parameters, then selecting the $\mathrm{CH}$ for the transmission processand their data. MATLAB programming platform is used to 
encode the SEECH and ZEEHC. The performance of ZEEHC is explained in terms of network lifetime and stability period against the SEECH protocol.The main objective of the ZEEHC is uniform energy distribution, ensuring that the network remains fully operational in the maximum amount of time. WSN's main goal is to field sensors, sensor node that works when used with requested information as equal to the energy consumption of each sensor is a fully functioning network is essential.

The proposed scheme is zone based energy efficiency protocol that improve network lifetime in three different scene1, scene 2,scene 3 with deployment shown in Figures 3-5 respectively.The performance of ZEEHC is compared to SEECH protocol in three scenarios. Table 1 defines the parameters of proposed scenes in details. A comparison of the number of alive nodes in scene 1, scene 2, and scene 3 for SEECH Protocol and ZEEHC Protocol is presented. The CHdistribution is needed in clustered WSN application.

Table1 Simulationparameters used for ZEEHC

\begin{tabular}{llll}
\hline Parameter & Scene 1 & Scene 2 & Scene 3 \\
\hline Area & $(0-0),(100-100)$ & $(0-0),(100-100)$ & $(0-0),(100-100)$ \\
Number of nodes, $N$ & 100 & 400 & 1000 \\
Initial energy of normal node, $E_{0}$ & $0.5 \mathrm{~J}$ & $0.5 \mathrm{~J}$ & $1 \mathrm{~J}$ \\
Transmission range within cluster & & $87 \mathrm{~m}$ & \\
Radio electronics energy, $E_{T x}=E_{R x}$ & & $50 \mathrm{~nJ} / \mathrm{bit}$ & \\
Radio amplifier energy, $f_{f}$ & & $10 \mathrm{p} / \mathrm{bit} / \mathrm{m}^{2}$ & \\
Radio amplifier energy, $\varepsilon_{t w o}$ ray_amp & & $0.0013 \mathrm{p} / \mathrm{bit} / \mathrm{m}^{4}$ & \\
Energy for data-aggregation, $E_{D A}$ & $5 \mathrm{~nJ} / \mathrm{bit} / \mathrm{signal}$ & \\
Data packet size & & $4000 \mathrm{bits}$ & \\
\end{tabular}

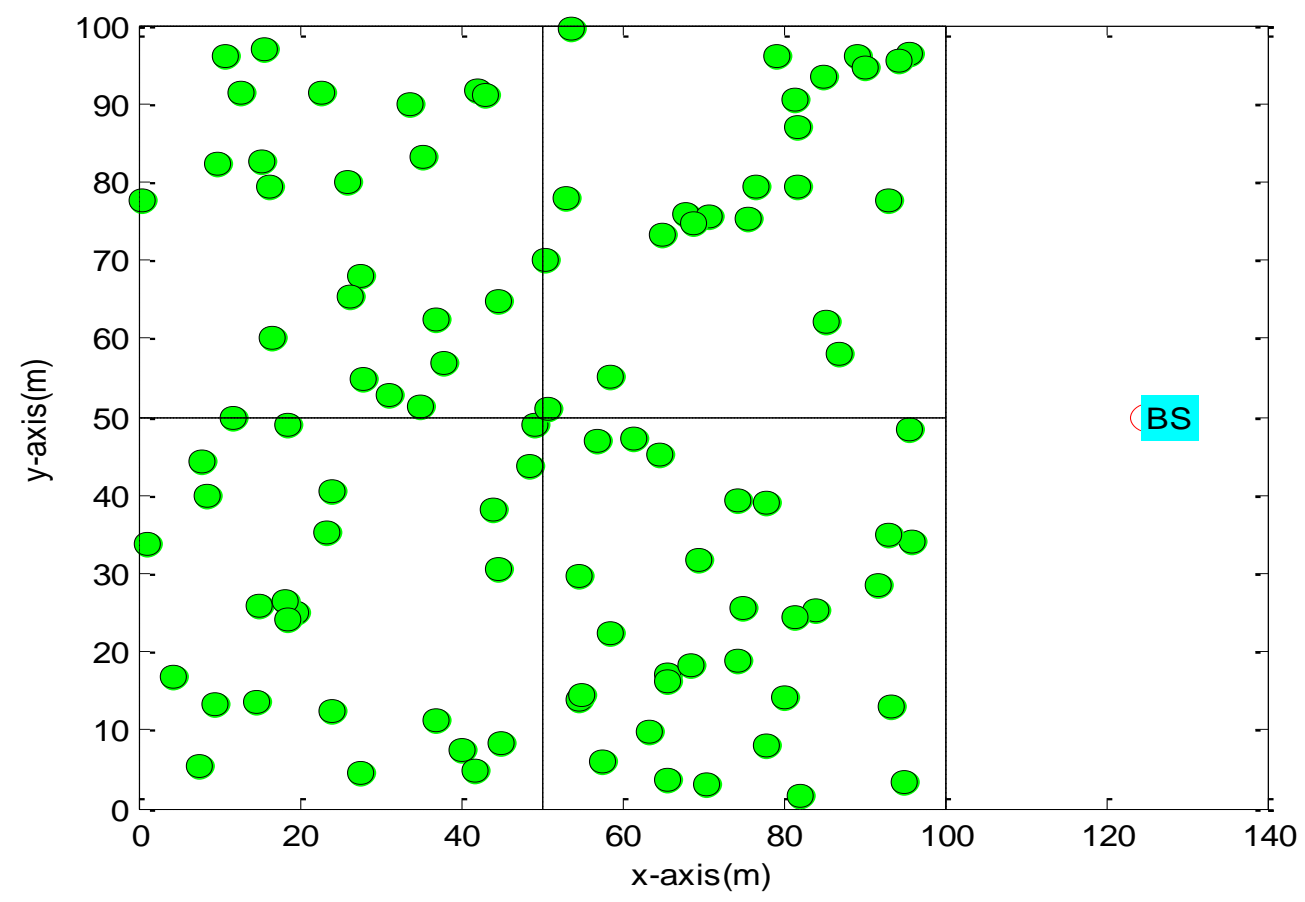

Fig. 3 Deployment of ZEEHC for (Scene 1) 100 nodes 


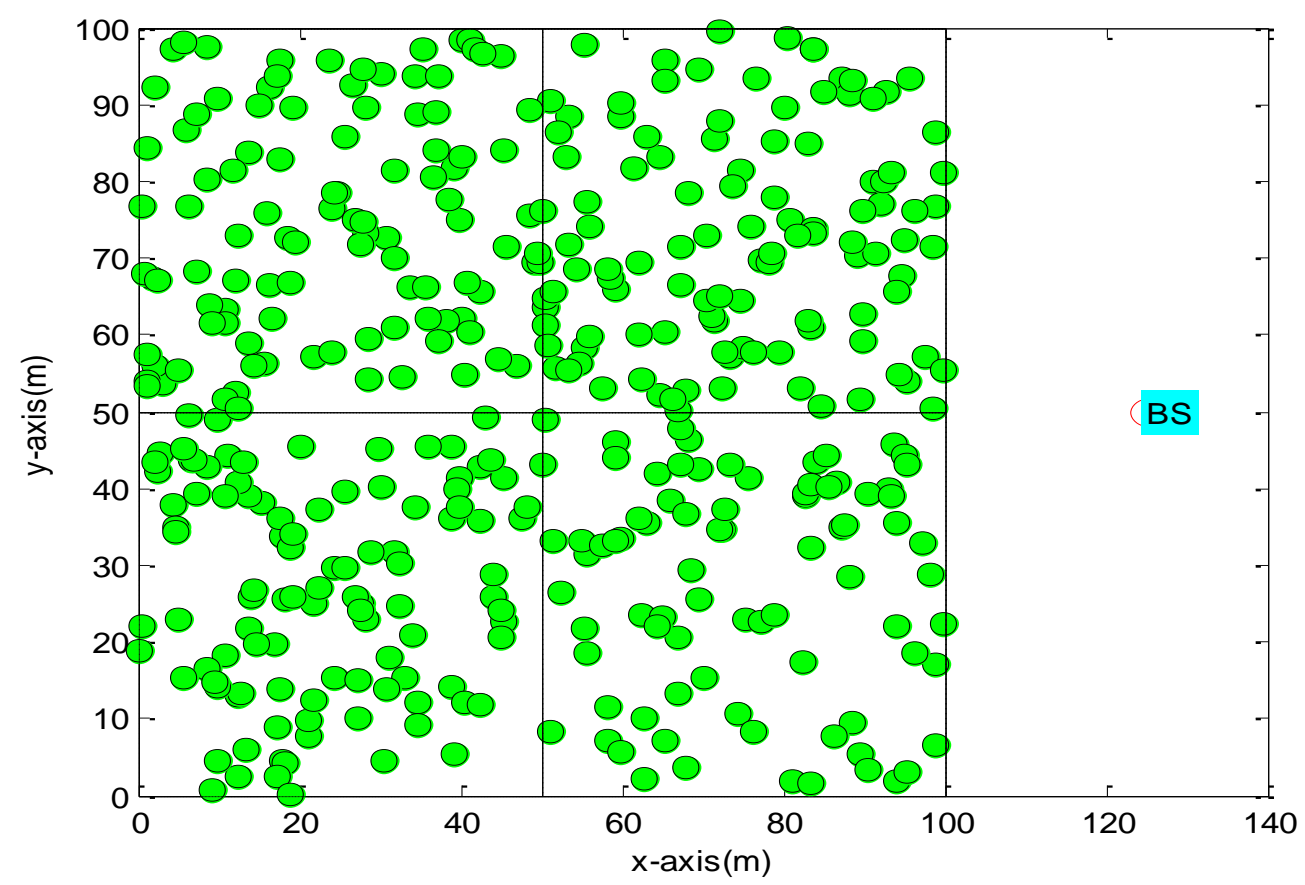

Fig. 4 Deployment for ZEEHC (Scene) 2 for 400 nodes

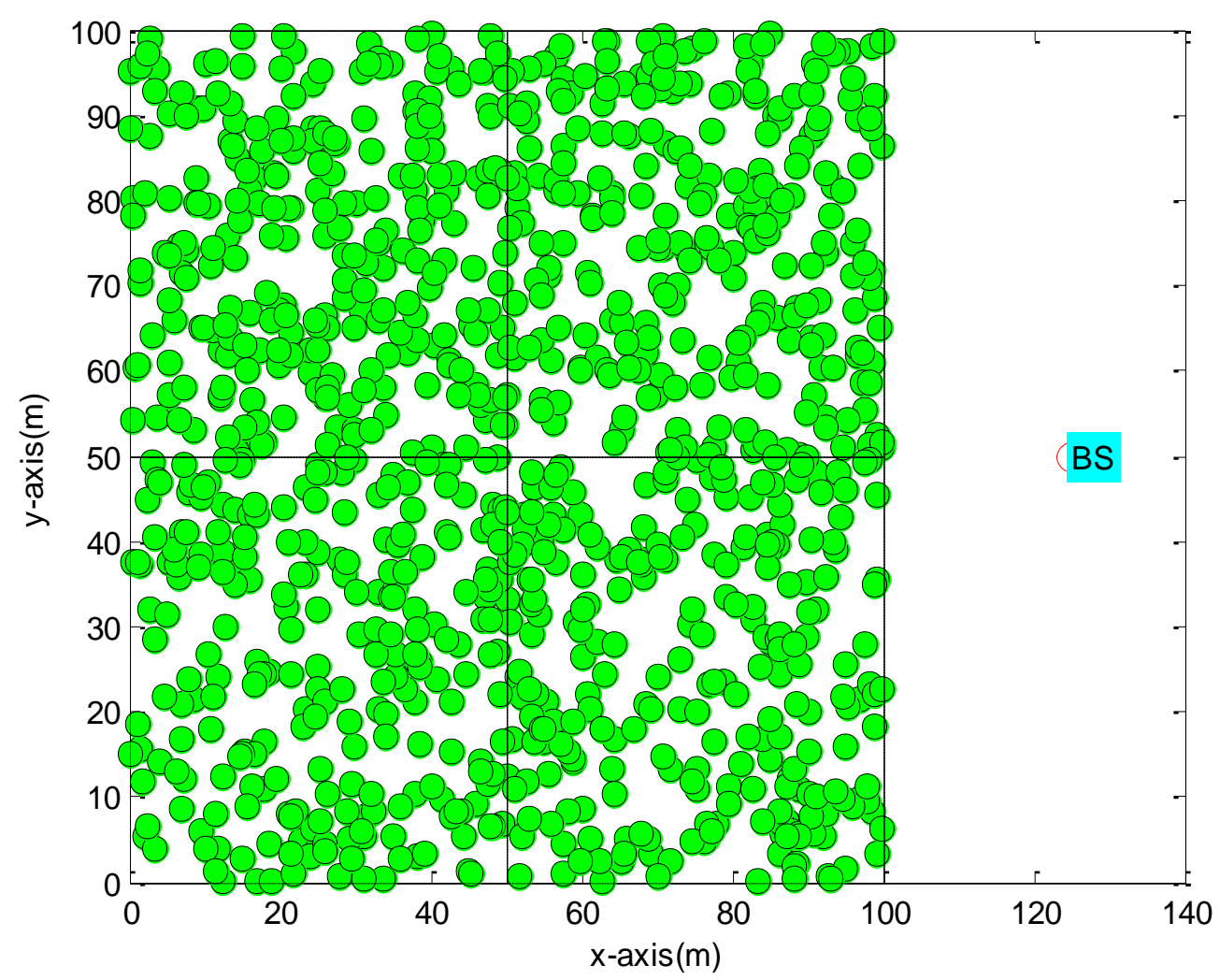

Fig. 5 Deployment for ZEEHC (Scene 3) 1000 nodes

In ZEEHC protocol multihop communication scheme is used. In this scheme cluster member send their data to their nearest $\mathrm{ZH}$ (denoted in blue color) in which $\mathrm{ZH}$ is elected based on remaining energy and the minimum distance from the BS. After receiving data from cluster member, $\mathrm{ZH}$ aggregate the data and send to Zone relay node (denoted in red color), then relay node transmit the data to BS using multihop to other relays which is shown in figures 6-8. 


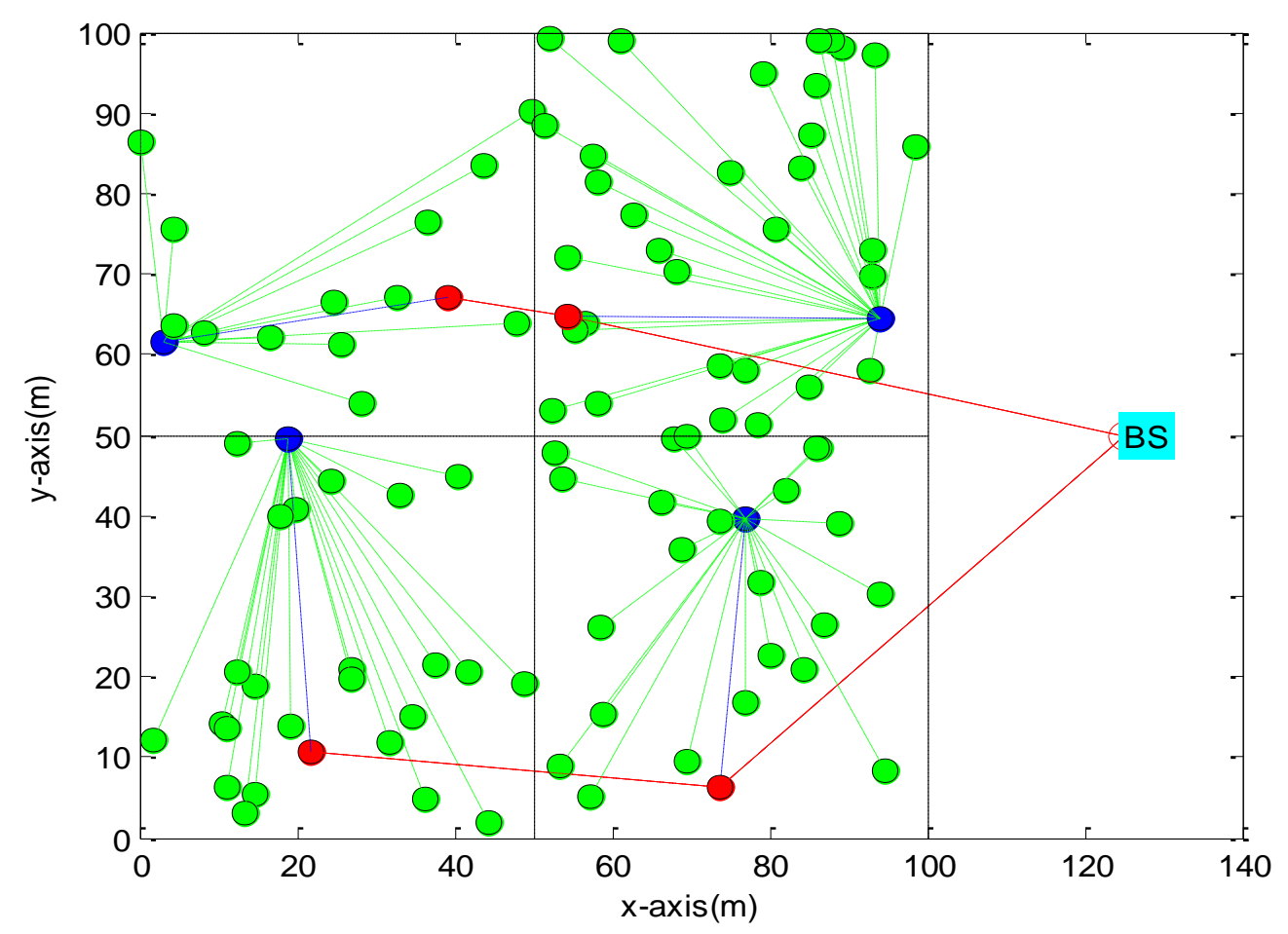

Fig. 6Multihop communication in ZEEHC (Scene 1) 100 nodes

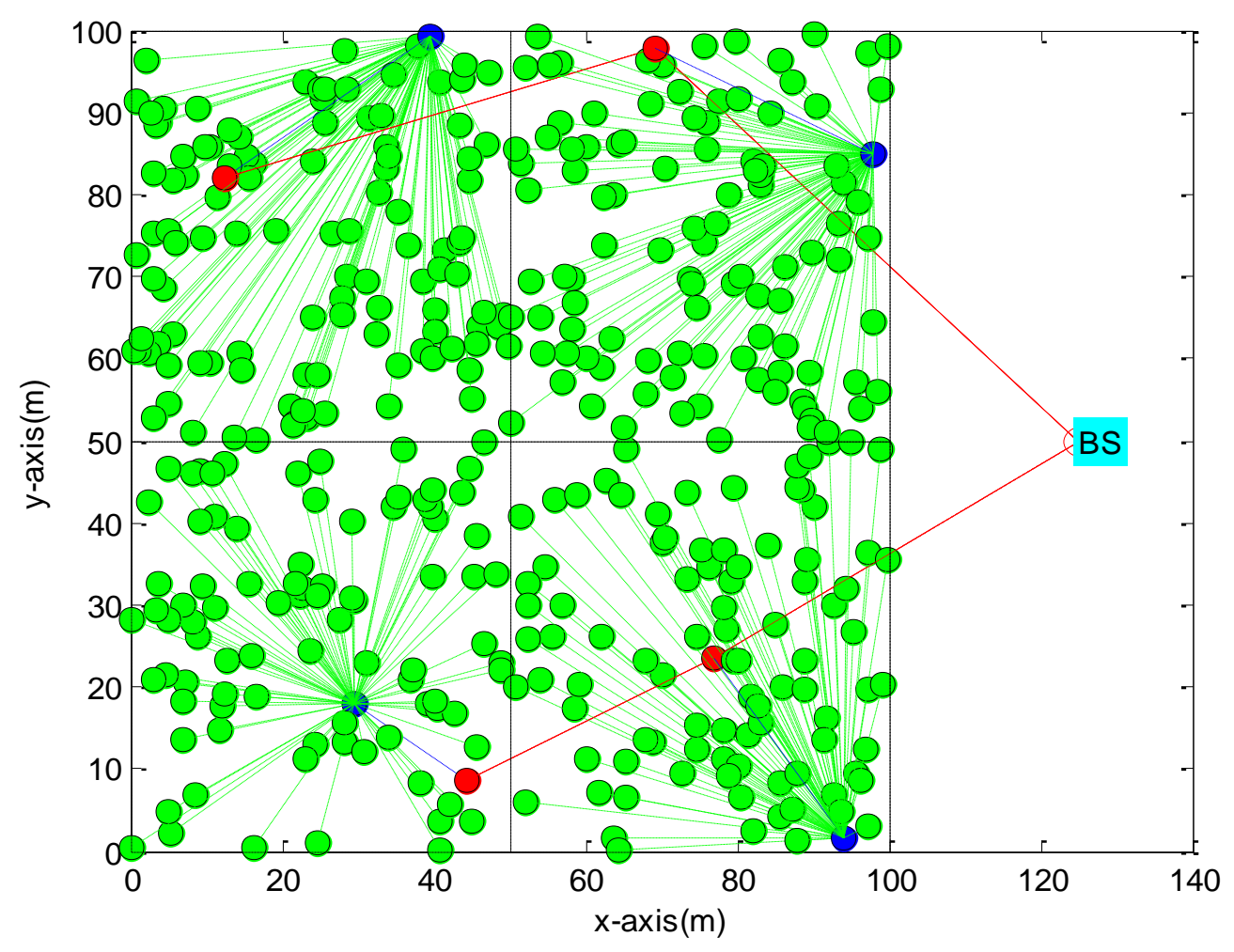

Fig. 7Multihop communication in ZEEHC (Scene 2) 400 nodes 


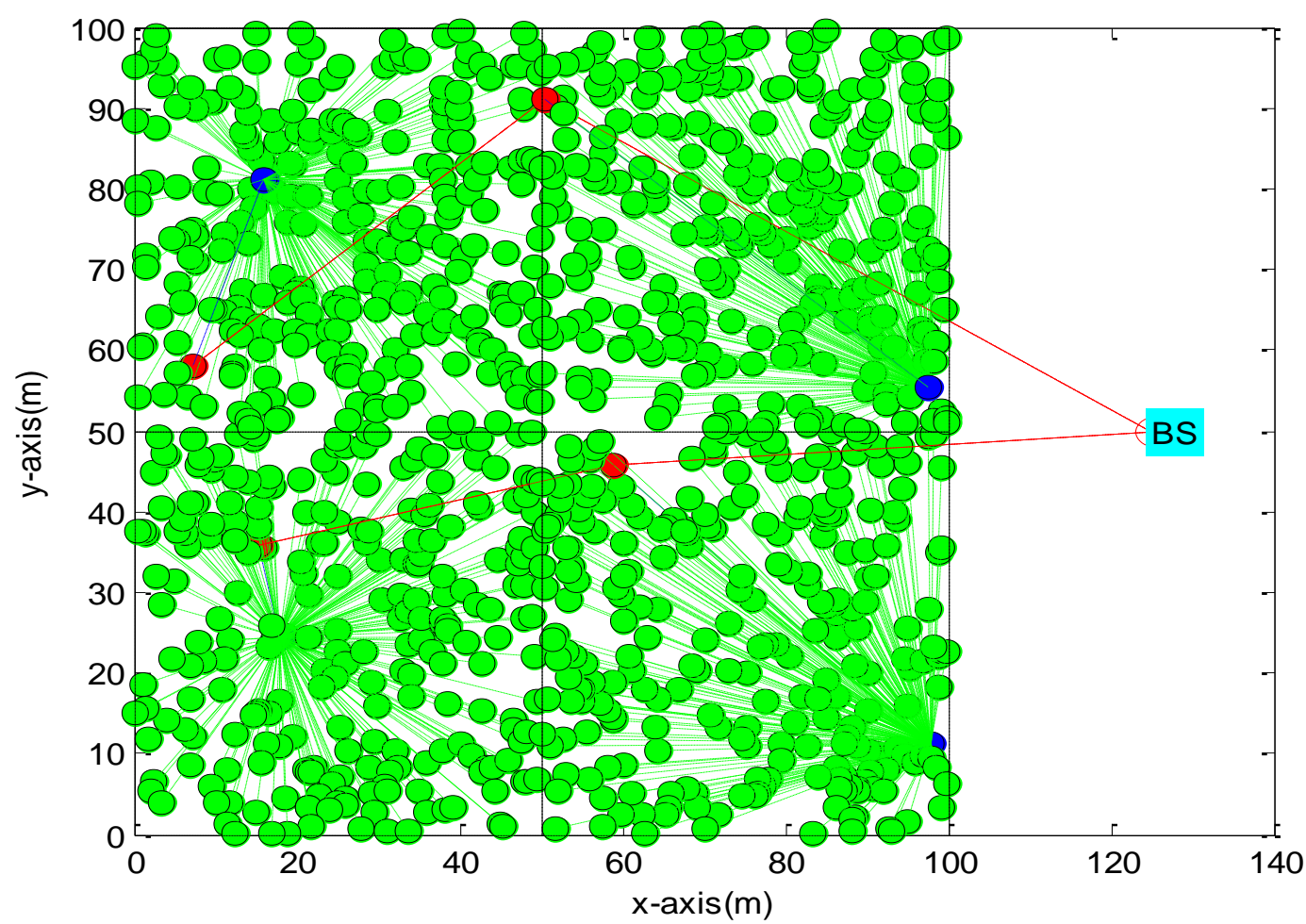

Fig. 8Multihop communication in ZEEHC (Scene 3) 1000 nodes

A comparison of the number of alive nodes in scene 1, scene 2, and scene 3 for SEECH Protocol and ZEEHC Protocol is presented.Alive nodes are those nodes whose battery is not completely depleted. The evaluated results are shown in Figures 9-11 for different scenarios.

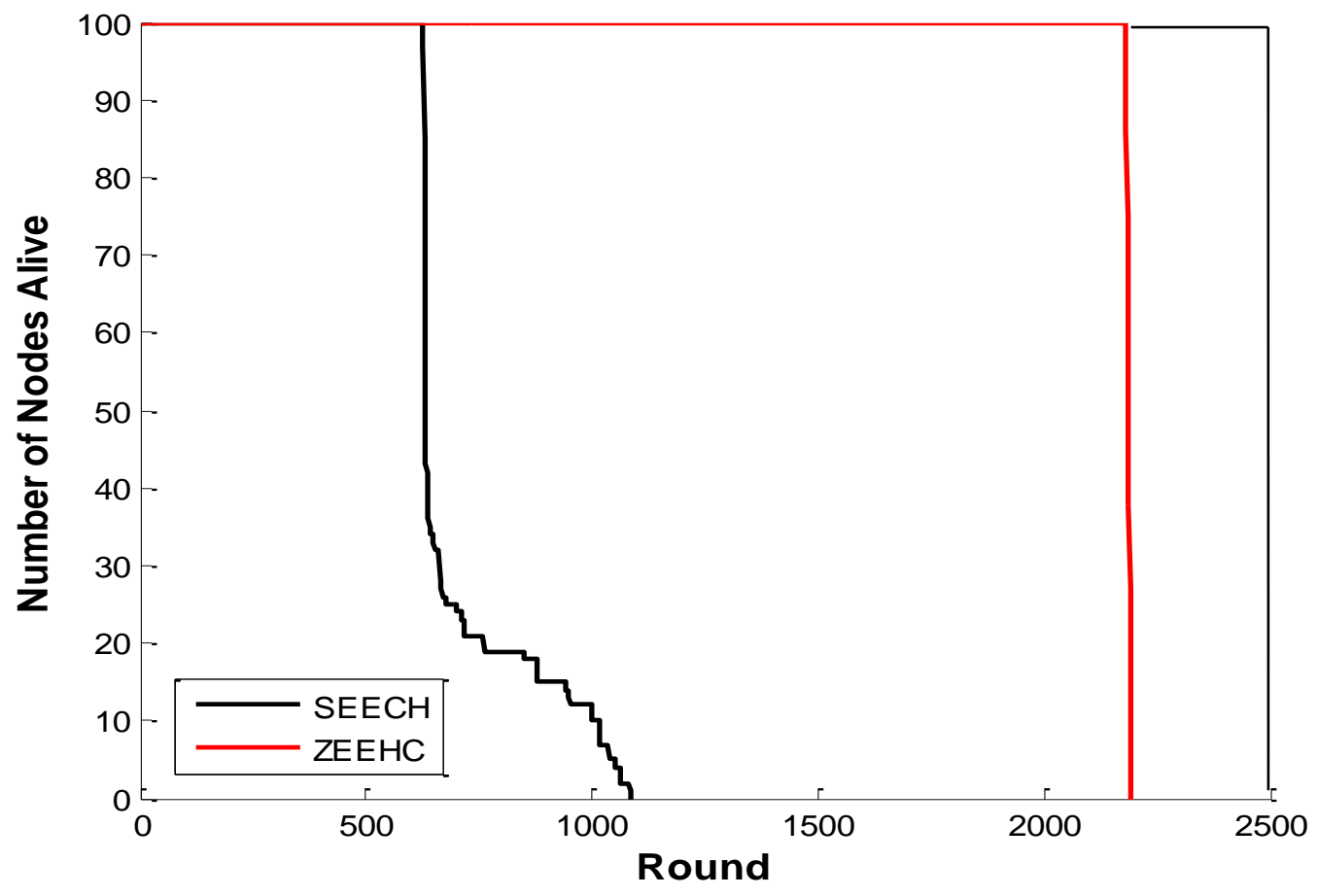

Fig.9No. of alive nodes for (scene 1)100 nodes 


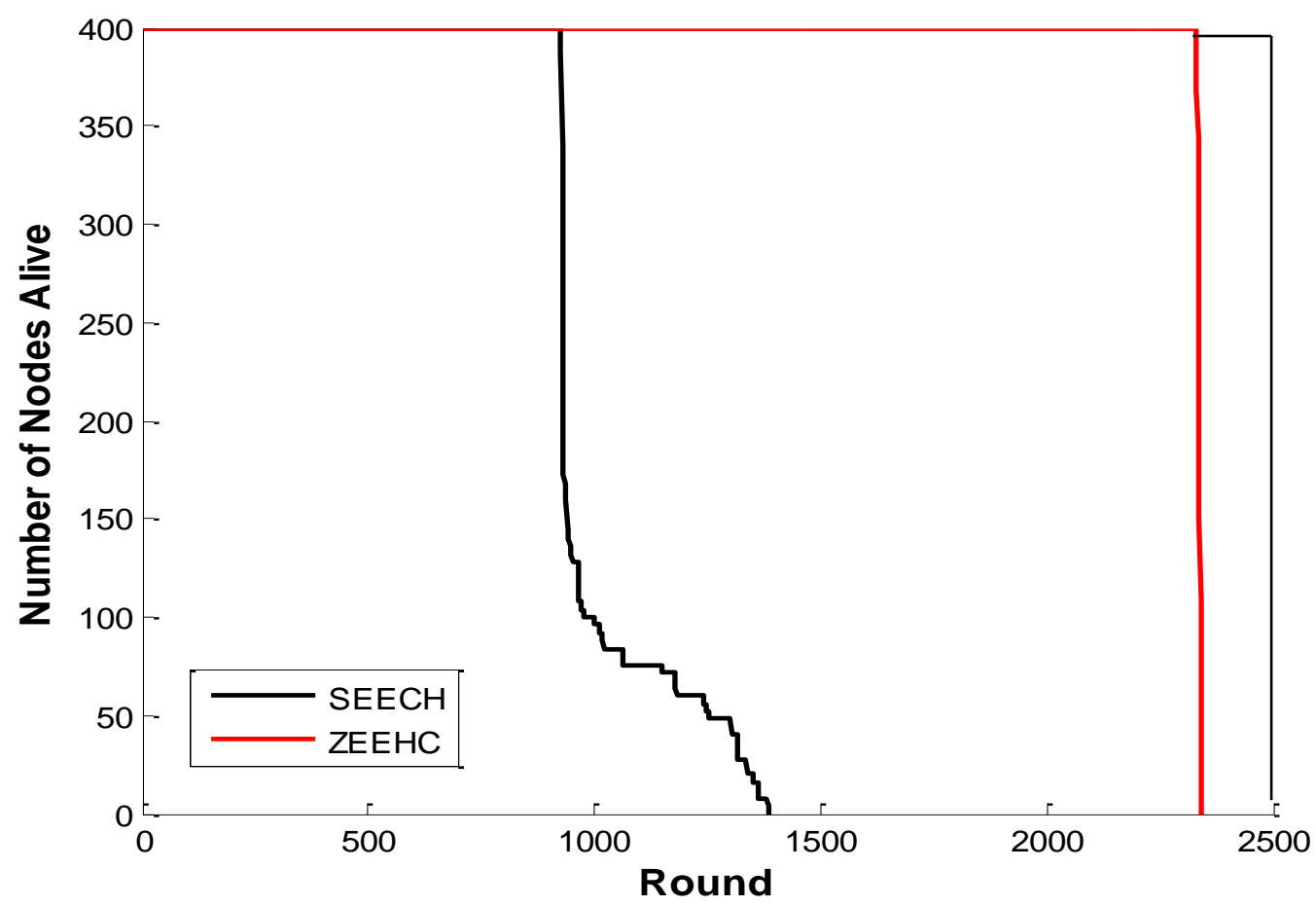

Fig. 10 No. of alive nodes for (scene 2) 400 node

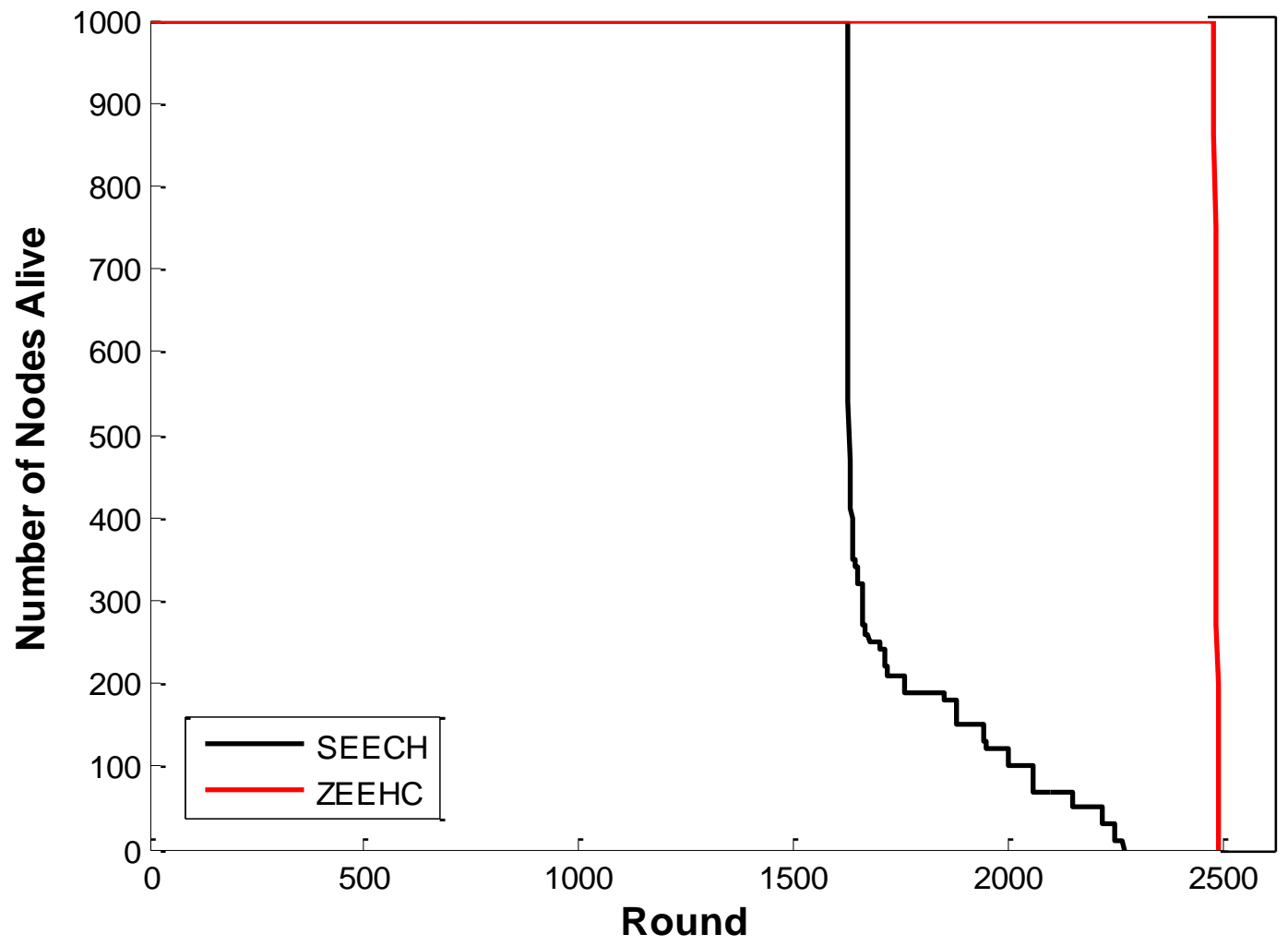

Fig. 11No. of alive nodes for (scene 3) 1000 nodes

The CHdistribution is needed in clustered WSN application. The intra-cluster delay is maximum and proportionate to the size of the biggest cluster therefore maintaining cluster avoid increase of delay and avoid large-distant communication between $\mathrm{CH}$ and cluster member. To measure the quality of distribution for $\mathrm{CHs}$ we use energy dissipation as shown in Figures 12-14. 


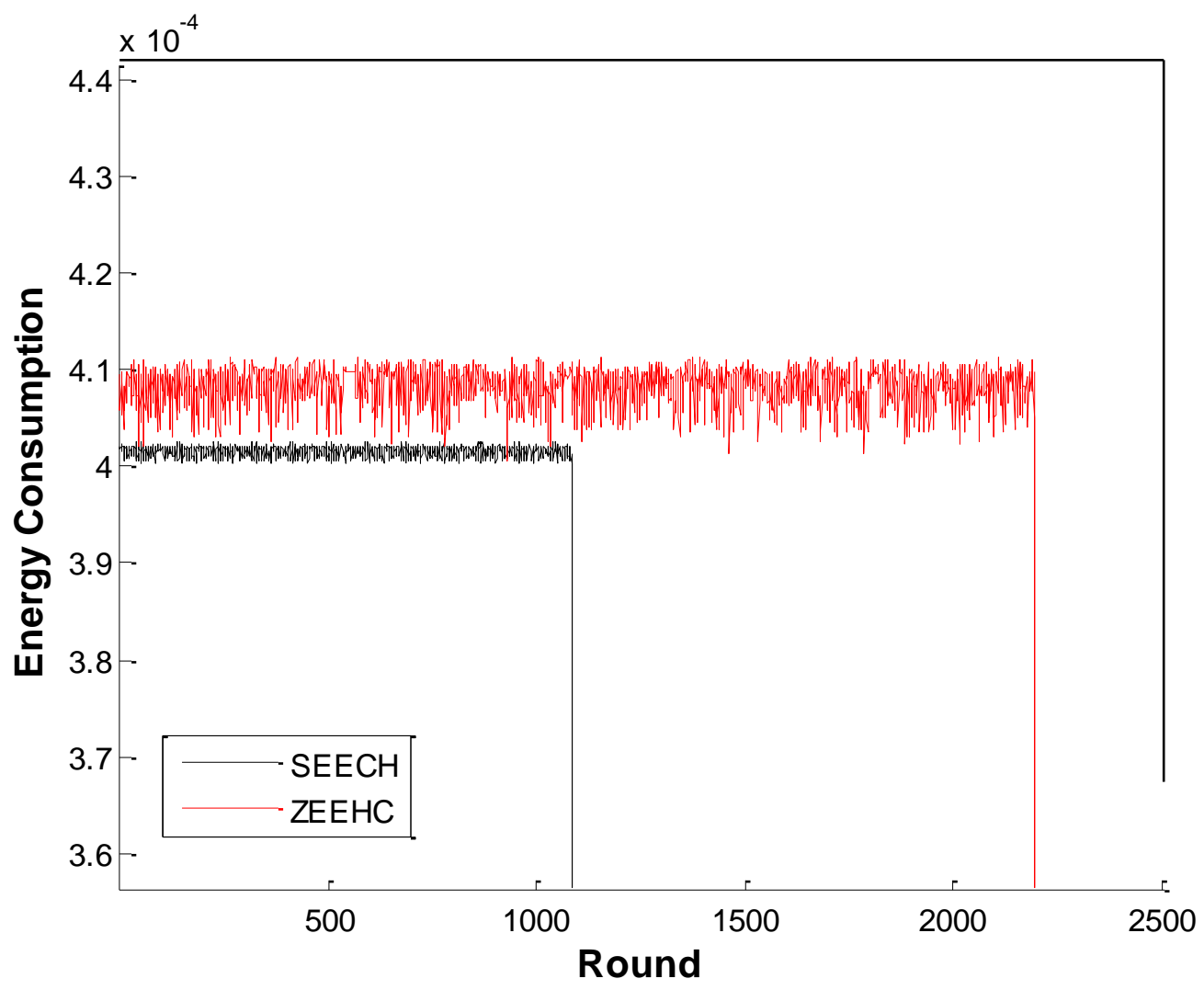

Fig.12Energy Dissipation for scene 1(100 nodes)

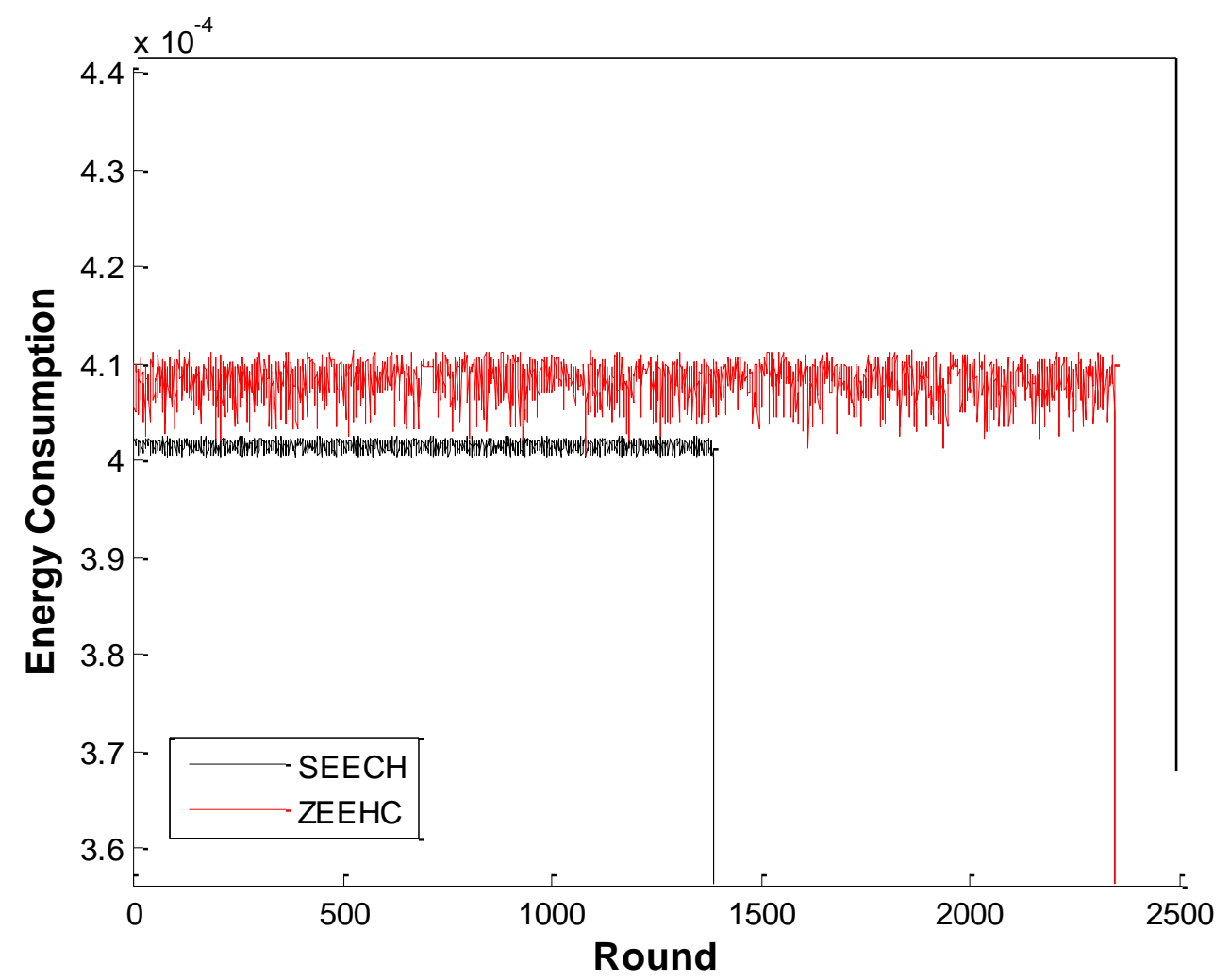

Fig. 13Energy Dissipation for scene 2(400 nodes) 


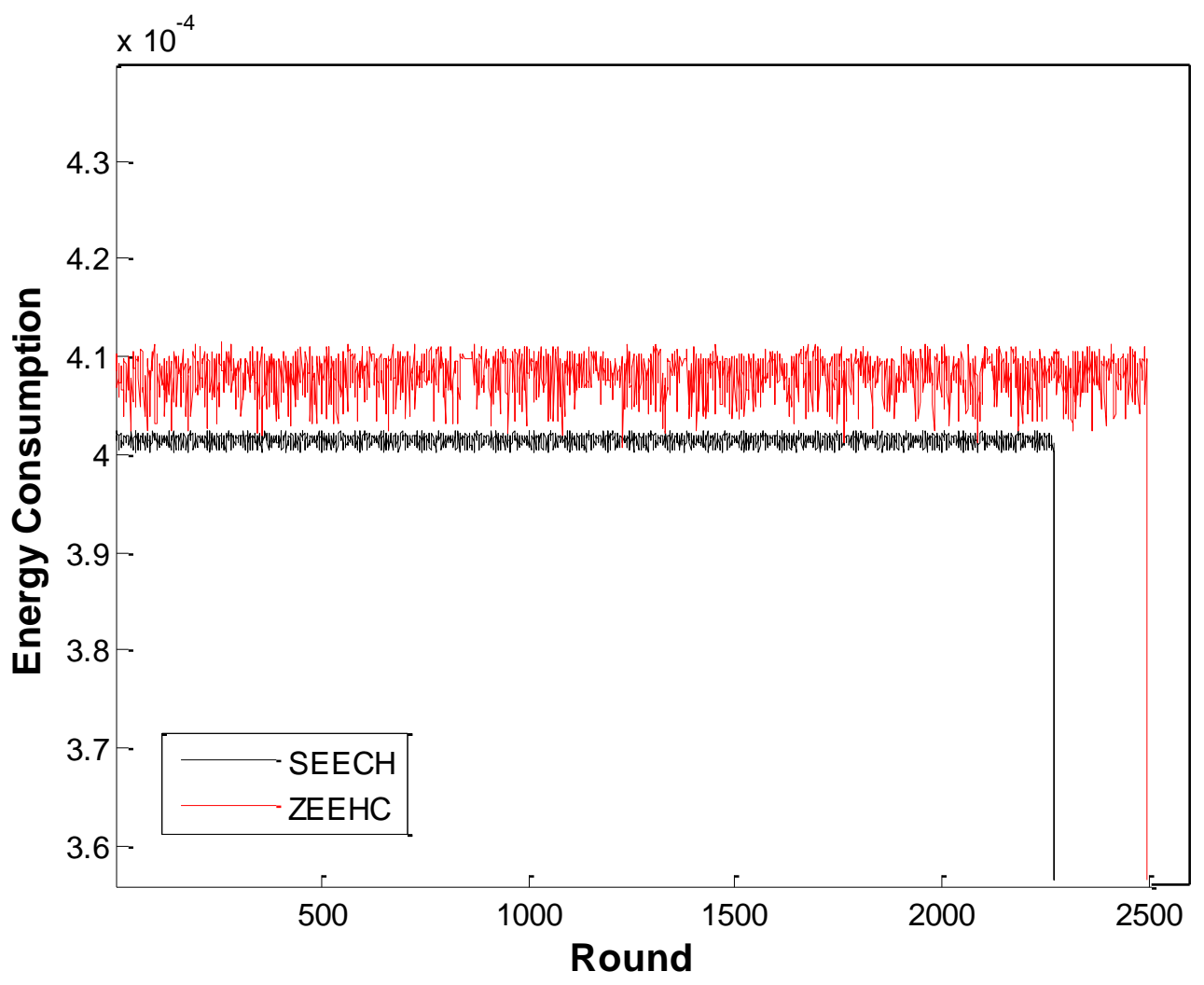

Fig. 14Energy Dissipation for scene 3(1000 nodes)

We also compare the performance of ZEEHC against SEECH in terms of the first node dies (FND), the average node dies (AND), the last node dies (LND). Table 2 shows results on the basis of initial energy of nodes.In research work, three cases are considered for simulations of our proposed protocol ZEEHC which are given below:

Case 1:100 nodes and location of sink $(50,175)$

Case 2:400 nodes and location of sink $(50,200)$

Case3:1000 nodes and location of sink $(100,350)$

Table 2Comparison between the lifetime of SEECH and ZEEHC in different scenes

\begin{tabular}{cccccc}
\hline Scene & Initial Energy & Protocol & FND & AND & LND \\
\hline $\begin{array}{c}\text { Scene } 1 \\
(100 \text { Nodes })\end{array}$ & & SEECH & 832 & 1248 & 1358 \\
\hline $\begin{array}{c}\text { Scene } 2 \\
(400 \text { Nodes })\end{array}$ & $0.5 \mathrm{~J}$ & ZEEHC & 1163 & 2066 & 2320 \\
\hline $\begin{array}{c}\text { Scene 3 } \\
(1000 \text { Nodes })\end{array}$ & SEECH & ZEEHC & 938 & 1347 & 1456 \\
& & SEECH & 1568 & 2105 & 2406 \\
\hline
\end{tabular}

When any node in the network is dead, it is no longer the part of that network. This implies that if a dead node occurs in early rounds, it will affect the network performance. This may also lead towards the early dead of all the nodes in the network. In this simulation we have observed the first dead node by keeping the base station position at $(100,200)$ with 4000 bits packet size. Table 2 shows the values and Fig. 15-17 conclude that ZEEHC is better compared to SEECH protocol.

Case1:In this, no. of nodes is 100 varying against no of rounds as shown in figure 15 . Initial energy is $0.5 \mathrm{~J}$. The number of nodes in the network was varied and their effect on lifetime was considered. 


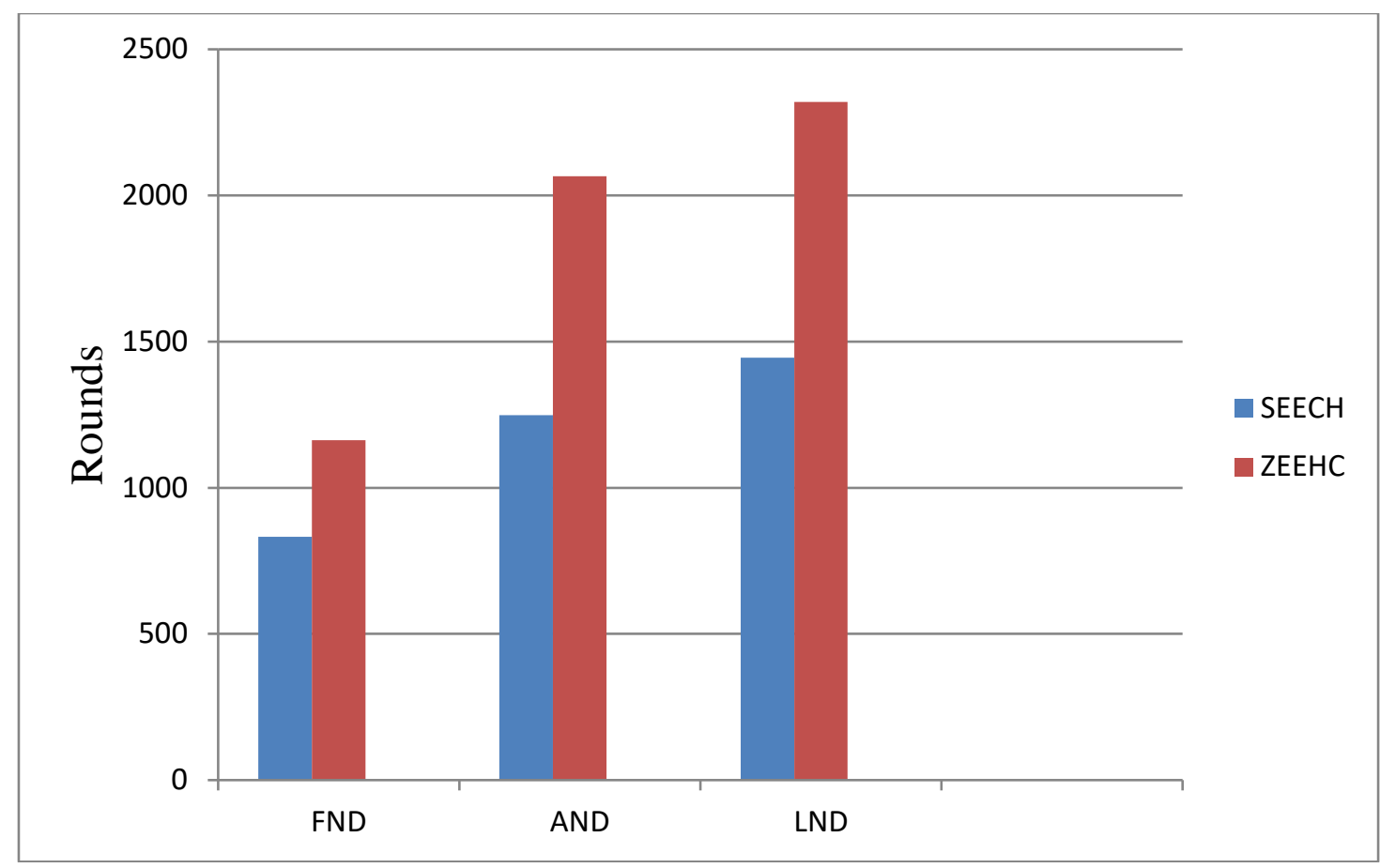

Fig. 15Performance results for FND, AND, LND for Scene 1

Case2:In this, no. of nodes is 400 varying against no of rounds as shown in figure 16 . Initial energy is $0.5 \mathrm{~J}$. The number of nodes in the network was varied and their effect on lifetime was considered.

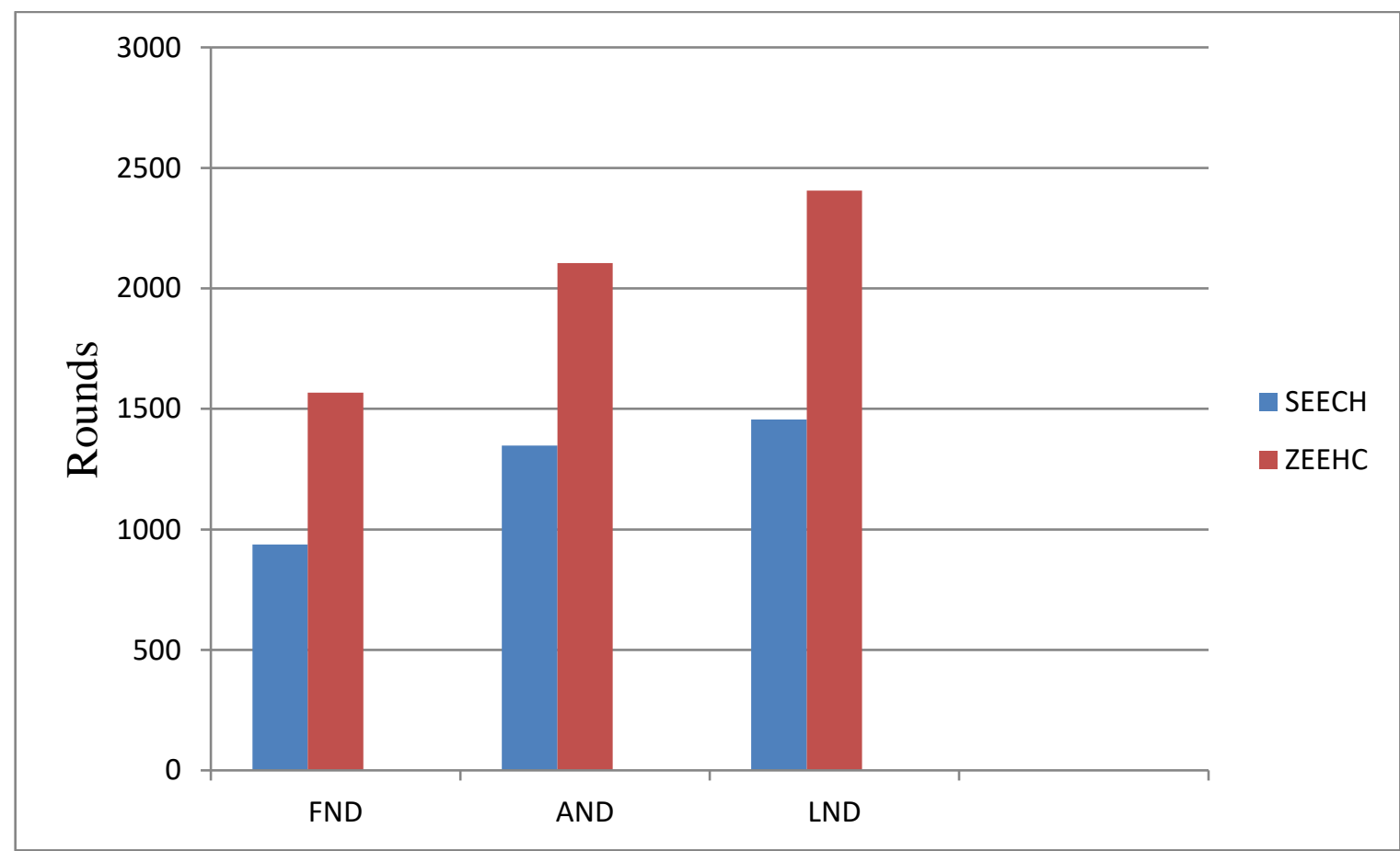

Fig. 16 Performance results for FND, AND, LND for Scene 2

Case3:In this, no. of nodes is 400 varying against no of rounds as shown in figure 17. Initial energy is $0.5 \mathrm{~J}$. The number of nodes in the network was varied and their effect on lifetime was considered. 


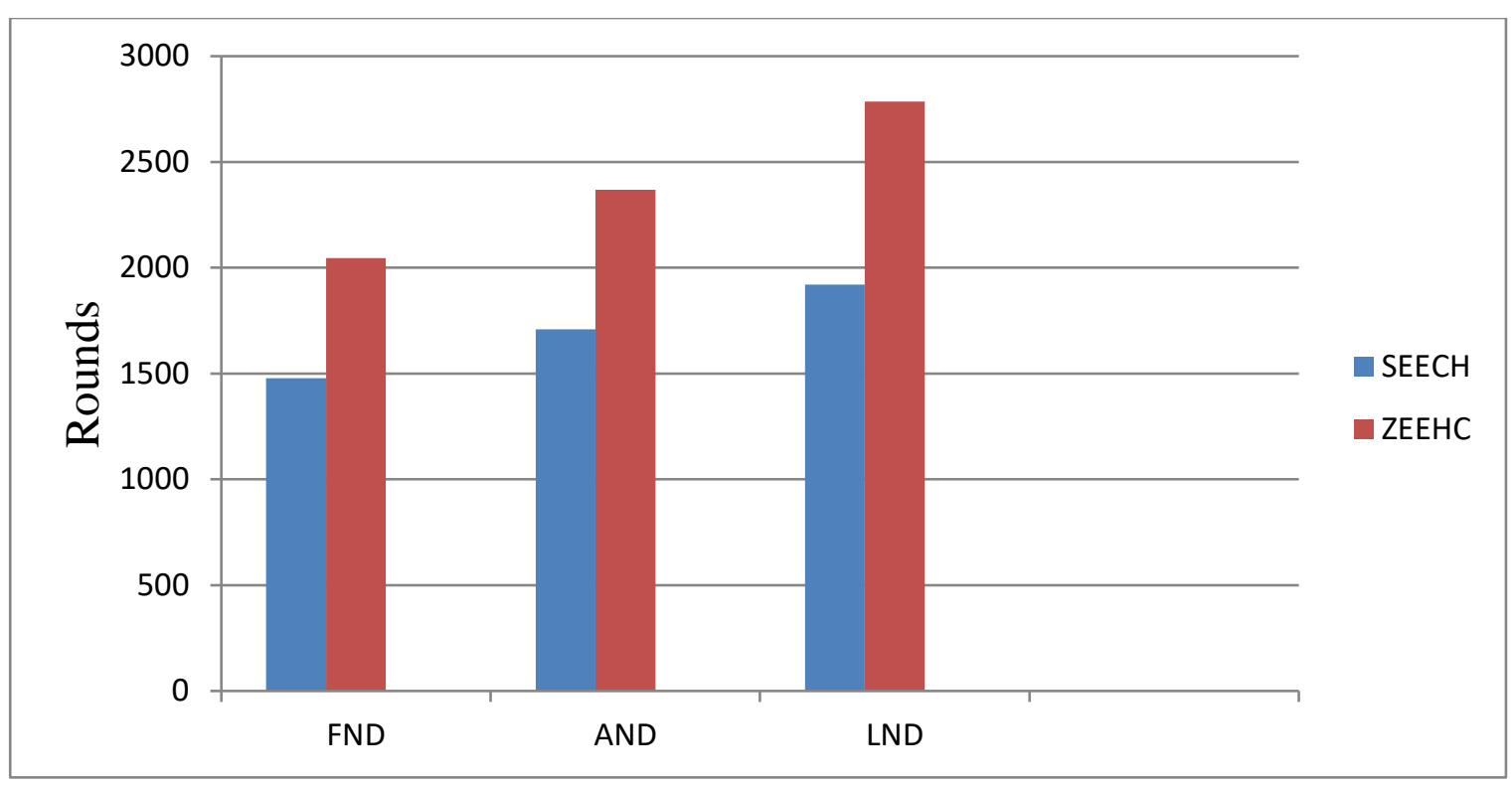

Fig. 17 Performance results for FND, AND, LND for Scene 3

\section{Conclusion and Future work}

In Smart Space and Extreme environment energy-aware distributed sensor monitoring and data clustering is relevant to save power. The important design issues in routing protocols are energy utilization [24]and network lifetime. A major challenge in routing protocol is to achievehigh stability period in order to preserve the coverage area.A zone based energy efficient hierarchy Protocol (ZEEHC) clustering is proposed in that the member nodes, cluster heads and relay are categorized differently within the network. ZEEHCdepicts a simple message of low overhead approach for periodic information accumulated applications in harsh and remote environments that extend network lifetime under three scenarios. The simulation outcomes showed that ZEEHC protocol achieves better lifetime than SEECH. Simulation results show that ZEEHC can be used for large scale networks. Adjacent improvement can be possible by considering nature inspired algorithm for successful delivery of data.

\section{References}

1. K. V. Shukla, Chandaben Mohan Bahi, "Energy efficient routing protocol LEACH for wireless sensor network”, IEEE Communications Surveys, vol.15,no.2, pp. 551-591, 2013.

2. W. B.Heinzelman, A. P. Chandrakasan and H. Bala krishnan, "An application-specific protocol architecture for wireless micro sensor networks," IEEE Transactions on Wireless Communication., vol. 1, no. 4, pp. $660-670$.

3. V. Loci, G. Moabite, and S. Marino, "A two-level hierarchy for low energy adaptive clustering hierarchy," IEEE Transaction Parallel Distributed System, vol. 3, no.2, pp. 1809-1813.

4. Chi-Stun Cheng, C. K. Toe and F. C. M. Lau, "A clustering algorithm for wireless sensor networks based on social insect colonies," IEEE Sensors Journals, vol. 11, no. 3, pp. 711-721, 2011.

5. N. Dimokas, D. Katsaros and Y. Manolopoulos, "Energy-efficient distributed clustering in wireless sensor networks," IEEE Transactions on Parallel and Distributed Systems, vol. 70, no. 4, pp. 371-385, 2010.

6. N. A. Pantazis, S. A. Nikolakakos, and D. D. Vergados, "Energy-efficient routing protocols in wireless sensor networks: A survey, " IEEE Communication Surveys, vol. 15, no. 2, pp. 551-591, 2013.

7. Asaduzzaman and H. Y. Kong, "Energy efficient cooperative LEACH protocol for wireless sensor networks," Journal of Communications and Networks, vol. 12, no. 4, pp. 358-365, 2010.

8. P. Gun, T. Jiang, K. Zhang, and H.-H. Chen, "Clustering algorithm in initialization of multi-hop wireless sensor networks," IEEE transactions on Wireless Communications, vol. 8, no. 12, pp. 5713-5717,2009.

9. Chi-Stun Cheng and F. C. M. Lau, "An energy-aware scheduling scheme for wireless sensor networks," IEEE Transaction wireless Technology, vol. 59, no. 7, pp. 3427-3444, 2010. 
10. Ossama Younis and Sonia Fahmy,“ HEED: A Hybrid, Energy-Efficient, Distributed Clustering Approach for Ad-hoc Sensor Networks," International Symposium on Parallel Architectures, Algorithms, and Networks (I-SPAN), vol. 1, no. 4, pp. 660-670, 2002.

11. M. Pope, O. Protean and A. S. Pope, "A classification of solutions for the energy limitation in wireless sensor networks," 9th IEEE International Conference Computer Cyber (ICCC), vol.11,no.3,pp. 293-297.

12. S. Lin, J. Zhang, G. Zhou, J. A. Stankovic and T. Hein, "ATPC: Adaptive transmission power control for wireless sensor networks," $4^{\text {th }}$ International Conference Embedded Network Sensor System, vol.13, no. 2, pp. 223-236, 2006.

13. S. H. Kang and T. Nguyen, "Distance based thresholds for cluster head selection in wireless sensor networks," IEEE Communication letters, vol. 16, no. 9, pp. 1396-1399, 2012.

14. N.Ahmed, .S.Kanhere, S.Jha, "The Holes Problem in Wireless Sensor Networks: A Survey," Mobile Computing and Communications Review, vol.1, no.2, pp. 1-14.

15. H. Wing, Y. Chen, H. Wu and G. Chen, "Correlated data gathering with double trees in wireless sensor networks," IEEE International Conference Wireless Mobile Computing, Networking Communication, vol. 12, no. 5, pp. 1147-1156, 2012.

16. M. Chu, H. Haussecker, and F. Zhao, "Scalable Information-Driven Sensor Querying and Routing for ad hoc Heterogeneous Sensor Networks, "The International Journal of HighPerformance Computing Applications, Vol. 16,No. 3,August 2002.

17. R. Sharma, B. S. Sohi, N. Mittal, "Zone-based Energy Efficient Routing protocol for Wireless Sensor Networks", Scalable Computing: Practice and Experience (SCPE), Special Issue on Opportunistic Network and its Security Challenges, Vol. 20, No. 1, pp 55-70, 2019.

18. Kaur, D., Aujla, G. S., Kumar, N., Zomaya, A. Y., Perera, C., \& Ranjan, R. (2018). Tensor-based big data management scheme for dimensionality reduction problem in smart grid systems: SDN perspective. IEEE Transactions on Knowledge and Data Engineering, 30(10), 1985-1998.

19. Gairola, P., Gairola, S. P., Kumar, V., Singh, K., \& Dhawan, S. K. (2016). Barium ferrite and graphite integrated with polyaniline as effective shield against electromagnetic interference. Synthetic Metals, 221, 326-331.

20. Mittal, N., Singh, U., \& Sohi, B. S. (2017). A novel energy efficient stable clustering approach for wireless sensor networks. Wireless Personal Communications, 95(3), 2947-2971.

21. Jindal, A., Aujla, G. S., \& Kumar, N. (2019). SURVIVOR: A blockchain based edge-as-a-service framework for secure energy trading in SDN-enabled vehicle-to-grid environment. Computer Networks, 153, 36-48.

22. Gupta, M. K., Mia, M., Singh, G., Pimenov, D. Y., Sarikaya, M., \& Sharma, V. S. (2019). Hybrid coolinglubrication strategies to improve surface topography and tool wear in sustainable turning of Al 7075-T6 alloy. The International Journal of Advanced Manufacturing Technology, 101(1-4), 55-69.

23. Garg, S., Singh, A., Kaur, K., Aujla, G. S., Batra, S., Kumar, N., \& Obaidat, M. S. (2019). Edge computingbased security framework for big data analytics in VANETs. IEEE Network, 33(2), 72-81.

24. Batoo, K. M., Kumar, G., Yang, Y., Al-Douri, Y., Singh, M., Jotania, R. B., \& Imran, A. (2017). Structural, morphological and electrical properties of $\mathrm{Cd} 2+$ doped $\mathrm{MgFe} 2-\mathrm{xO} 4$ ferrite nanoparticles. Journal of Alloys and Compounds, 726, 179-186.

25. Sharma, G., Sharma, S., \& Gujral, S. (2015). A novel way of assessing software bug severity using dictionary of critical terms. Procedia Computer Science, 70, 632-639. 